\title{
Critical Scrutiny of the Visualization and Detection of Oral Potentially Malignant Disorders by Chemiluminescent Illumination
}

\author{
Subashini Suyambukesan ${ }^{1 *}$, Gopuchandran Lenin Perumal ${ }^{2}$, \\ S. Shanmugam ${ }^{3}$ and Vishwanath Rangdhol ${ }^{4}$ \\ 'Department of Oral Medicine \& Radiology, Penang International Dental College, NB Tower, \\ 12000, Butterworth, Penang, Malaysia; drsuba.academics@gmail.com \\ 2Department of Periodontology, Penang International Dental College, NB Tower, \\ 12000, Butterworth, Penang, Malaysia; gopuchandranlenin@gmail.com \\ ${ }^{3}$ Department of Oral Medicine \& Radiology, Ragas Dental College, Chennai, \\ India; shanmugammds@gmail.com \\ ${ }^{4}$ Department of Oral medicine and Radiology, Indira Gandhi institute of Dental sciences, \\ Pillayarakuppam, Pondicherry, India; drvishident@gmail.com
}

\begin{abstract}
The aim of this study was to critically analyse the effectiveness of Vizilite in detecting Oral Potentially Malignant Disorders (OPMD). This study was conducted on 70 patients, among them, 50 were identified to have Oral Potentially Malignant Disorders and the remaining 20 subjects had no apparent lesions. Complete oral examination was performed for all the patients with normal incandescent light and Vizilite. All 50 cases with oral lesions underwent incision biopsy. The sensitivity and specificity of vizilite was investigated. Vizilite examination was $100 \%$ sensitive and specific for leukoplakia. But, the examination was not quite sensitive for lichen planus and oral submucous fibrosis. No additional lesions were detected in normal subjects, no extra focal areas of lesions were detected in OPMD patients and there was no change in the biopsy site after vizilite examination. Oral Medicine specialists can diagnose the presence or can predict the progress of any Oral Potentially Malignant Disorders by conventional screening and complete examination. No additional benefits were obtained from the use of Vizilite.
\end{abstract}

Keywords: Chemiluminescent Illumination, Oral Cancer, Oral Potentially Malignant Disorders, Vizilite

\section{Introduction}

Oral Cancer, a preventable disorder is the sixth most common cancer reported worldwide ${ }^{1}$. Though the mortality is comparatively lesser than other cancers, its morbidity is significant with more impact on the psychological perspective. There are many proposed aetiologies for oral cancer, of which tobacco abuse is of prime concern ${ }^{2,3}$.

Oral potentially malignant disorders encompass a variety of lesions ${ }^{4}$. Oral premalignant lesions can be defined as altered epithelial lesions which have an increased likelihood of progressing to squamous cell carcinoma. The taxonomy, natural history and prognostic value of this group of lesion was reviewed at an expert workshop held in London in $2005^{5}$. The group suggested that the dissimilarity between potentially malignant lesions and conditions should be abandoned in favour of a common terminology of Oral potentially malignant disorders. Field cancerization is a concept introduced by Slaughter et al in 1953, which states that oral cancer can

${ }^{*}$ Author for correspondence 
develop in multifocal areas adjacent to the primary cancer regions where there are premalignant changes ${ }^{6}$.

The key role of dentists in identifying, preventing and treating these potentially malignant disorders are stressed in many literatures. Guggenheimer et $\mathrm{al}^{7}$ stated that diagnosis of early innocuous lesions by inexperienced clinicians is a difficult task. An epidemiological study for Oral Cancer done by $\mathrm{Rao}^{8}$ observed that it is important to undertake programs to prevent and control Oral Cancer by screening for early diagnosis. Chemiluminescent illumination is a method to detect dysplastic changes clinically as a chair side investigatory protocol ${ }^{9}$.

The objectives of this study was to critically analyse the effectiveness of chemiluminescent visualization in detecting lesions among patients with no clinically identifiable lesions and to appreciate its sensitivity and specificity in detecting dysplastic changes in patients with clinically identifiable oral potentially malignant disorders.

\section{Materials and Methods}

The diagnostic kit includes a complete diagnostic kit for standard clinical examination and Vizilite kit ${ }^{\oplus}$ manufactured by Zila Pharmaceuticals limited, Phoenix, AZ, USA which consists of $1 \%$ acetic acid solution, chemiluminescent light stick and a retractor.

Chemical luminescence was achieved by bending the capsule, whereby the products of the outer plastic capsule namely acetyl salicylic acid reacts with hydrogen peroxide present in the inner glass vial. The wavelength of the bluish white light emitted is $430-580 \mathrm{~nm}$. The light lasts for approximately 10 minutes after which it fades out.

\subsection{Inclusion Criteria}

1. All the patients irrespective of age, sex or socio economic background with the habit of cigarette smoking or use of tobacco in any forms.

2. Patients diagnosed clinically as having any one of the lesion- leukoplakia, erythroplakia, Oral Submucous fibrosis or oral lichen planus.

\subsection{Exclusion Criteria}

1. Patients without any deleterious habits of tobacco usage.

2. Patients with any systemic disorders or mentally unstable patients

3. Patients with dentures, sharp teeth and with parafunctional habits.
4. Lesions covered with clinically intact/ normal epithelium.

5. Patients with evidence of clinical lesions like Leukoedema, Linea alba buccalis or Fordyce`s granules.

6. Any sub mucosal swelling or mass or benign tumours.

7. Multiple lesions in a same patient were excluded.

8. Patients who are allergic to any ingredients of the vizilite kit.

\subsection{Study Sample}

The study was a hospital based cross sectional study. A total of 70 patients (males-59, females- 11) were subjected to chemiluminescent examination. All the 70 patients had history of tobacco usage.

\subsection{Methods}

All the patients were briefed about the procedure; consent obtained and was explained about the importance and significance of the study. Detail history was taken from the patient. Complete clinical examination was done with good illumination. Regional lymph nodes were examined. The clinical diagnosis was achieved based upon standard diagnostic protocols. Three oral diagnostic specialists examined the patients separately and the patients were considered for the study only if the same clinical diagnosis was arrived without any bias amongst the examiners. Among the total 70 patients examined, 20 had normal oral mucosa. The clinical diagnosis arrived for the remaining patients were as follows; 21- luekoplakias, 16- Oral Lichen Planus and 13- Oral Sub Mucous Fibrosis.

If a potentially malignant lesion was observed, its location, site, size, shape, surface, extent and colour was noted and digitally photographed. The lesion was palpated to know its consistency and tenderness. The lesion was checked for pseudo membrane and it was stretched out to rule the benign nature. Mouth opening was recorded. The suspicious sites for dysplasia were evaluated and potential biopsy sites were noted in a separate oral topography proforma.

Examination with vizilite was done according to the manufacturer's instructions. The patient was asked to rinse the mouth with plain water, then with $30 \mathrm{ml}$ of $1 \%$ acetic acid and expectorate it after 1 minute. The acetowhite areas were recorded and snapped. Vizilite capsule was activated and placed on the retractor. The lights in the 
clinics were dimmed and the oral cavity was completely examined. All the findings were recorded and photographed. Later the patient was asked to rinse the mouth with normal water and expectorate after 30 seconds.

The positive and negative areas were identified and incisional biopsy was done correlating with the positive site identified by Vizilite and negative cases were also biopsied for all the cases of OPMD. So, all the 50 lesions were biopsied. Patients with normally appearing mucosas were not biopsied for ethical considerations. There was no dysplastic sites detected by vizilite on these patients. The dysplasia was graded into mild, moderate and severe. The pathologists were blinded to the clinical diagnosis and also to that with Vizilite examination. There was no case were the proposed site of biopsy judged clinically was altered after Vizilite examination. The histopathological diagnosis was compatible with the clinical diagnosis. Table 1 depicts the differentiating appearance of the lesions with respect to site, size, shape and border distinctness between chemiluminescent visualization, acetic acid wash and under normal incandescent light.

\section{Results}

The results are depicted in Table 2 and 3. Of the 15 cases of Homogenous leukoplakia, 6 cases that were negative in
Vizilite examination were also negative for dysplasia and all the 9 positive cases were positive for dysplasia. It was noted that the 6 non- homogenous leukoplakia were positive with Vizilite and were also dysplastic. The sensitivity, specificity, positive and negative predictive values for each lesion are depicted in Table 3. False positive rate, false discovery rate, accuracy and F1 score which is a measure of test accuracy are illustrated in Table 4.

\section{Discussion}

Chemical luminescence is a process by which light energy is derived from chemical reactions. This technique is made use for the examination of the cervix and intra oral areas for detecting dysplastic lesions ${ }^{10}$. Vizilite is one such kit which makes use of chemiluminescence for detecting Oral Potentially Malignant Disorders. Many studies have been done in the past to illustrate the effectiveness of vizilite ${ }^{9,11-14}$. The manufacturer claims that ViziLite ${ }^{\circledast}$ TBlue $^{\otimes}$ is used to help oral healthcare professionals identify, evaluate, monitor and mark abnormal oral cell lesions suspicious for pathology including precancerous and cancerous cells that may be difficult to see during a regular visual examination (Assessed on 27th February 2014, as stated in Vizilite website http://www.zila.com/ vizilite/ ). The manufacturer of ViziLite is now marketing

Table 1. Comparison of the lesions between chemiluminescent examinations, after acetic acid wash and under incandescent light

\begin{tabular}{|c|c|c|c|}
\hline Criterias & $\begin{array}{c}\text { Lesion under } \\
\text { incandescent light }\end{array}$ & $\begin{array}{l}\text { Lesion after acetic acid } \\
\text { wash }\end{array}$ & $\begin{array}{c}\text { Appearance after vizilite } \\
\text { examination }\end{array}$ \\
\hline SITE & $\begin{array}{l}\text { Clinically identified by } \\
\text { Oral Medicine experts }\end{array}$ & $\begin{array}{l}\text { Remained the same } \\
\text { when compared with the } \\
\text { appearance of the lesion } \\
\text { under incandescent light. }\end{array}$ & $\begin{array}{l}\text { The appearance of the lesion was } \\
\text { enhanced. No new lesions were } \\
\text { detected }\end{array}$ \\
\hline SIZE & $\begin{array}{l}\text { Clinically identified by } \\
\text { Oral Medicine experts }\end{array}$ & $\begin{array}{l}\text { Remained the same } \\
\text { when compared with the } \\
\text { appearance of the lesion } \\
\text { under incandescent light }\end{array}$ & $\begin{array}{c}\text { There were no alterations in the } \\
\text { size of the lesion. }\end{array}$ \\
\hline SHAPE & $\begin{array}{l}\text { Clinically identified by } \\
\text { Oral Medicine experts }\end{array}$ & $\begin{array}{l}\text { Remained the same } \\
\text { but was more discrete } \\
\text { when compared with the } \\
\text { appearance of the lesion } \\
\text { under incandescent light }\end{array}$ & $\begin{array}{l}\text { Due to low visual light, the shape } \\
\text { was not the same as the optical } \\
\text { effect of the adjacent areas had } \\
\text { great influence }\end{array}$ \\
\hline $\begin{array}{l}\text { BORDER } \\
\text { DISTINCTNESS }\end{array}$ & $\begin{array}{l}\text { Clinically identified by } \\
\text { Oral Medicine experts }\end{array}$ & $\begin{array}{l}\text { Appears more distinct, } \\
\text { than examination under } \\
\text { incandescent light. }\end{array}$ & $\begin{array}{l}\text { Border was more clear and } \\
\text { distinct than under incandescent } \\
\text { light, as the reflectiveness of the } \\
\text { positive lesions was less. Borders } \\
\text { were less distinct than after acetic } \\
\text { acid wash. }\end{array}$ \\
\hline
\end{tabular}


Table 2. Result with chemiluminescent examination and histopathological diagnosis

\begin{tabular}{|c|c|c|c|c|c|c|c|c|c|}
\hline \multicolumn{2}{|c|}{ Clinical diagnosis } & \multirow{4}{*}{$\begin{array}{c}\text { Male } \\
13\end{array}$} & \multirow{4}{*}{$\begin{array}{c}\text { Female } \\
2\end{array}$} & \multirow{3}{*}{$\begin{array}{l}\text { Chemi } \\
\text { Pos } 9\end{array}$} & \multicolumn{5}{|c|}{ Histopathological diagnosis } \\
\hline & & & & & \multirow{2}{*}{$\begin{array}{c}\mathrm{HK} / \mathrm{o} / \mathrm{f} \\
-\end{array}$} & \multirow{2}{*}{$\frac{\mathrm{CiS}}{-}$} & \multirow{2}{*}{$\frac{\text { MD }}{5}$} & \multirow{2}{*}{$\frac{\text { MoD }}{3}$} & \multirow{2}{*}{$\frac{\text { SD }}{1}$} \\
\hline$\stackrel{5}{2}$ & Homogenous - 15 & & & & & & & & \\
\hline$\hat{0}$ & & & & Neg 6 & 6 & - & - & - & - \\
\hline 空. & Non & 5 & 1 & Pos 6 & - & 1 & 1 & 1 & 3 \\
\hline$\stackrel{\overparen{N}}{\ominus}$ & Homogenous-6 & & & Neg 0 & - & - & - & - & - \\
\hline \multirow{6}{*}{$\begin{array}{l}\text { OLP } \\
16\end{array}$} & Reticular- 8 & 7 & 1 & Pos 3 & 2 & - & 1 & - & - \\
\hline & & & & Neg 5 & 5 & - & - & - & - \\
\hline & Erosive- 5 & 3 & 2 & Pos 2 & 1 & - & 1 & - & - \\
\hline & & & & Neg 3 & 2 & - & - & 1 & - \\
\hline & Reticular+ & 2 & 1 & Pos 0 & - & - & - & - & - \\
\hline & atrophic- 3 & & & Neg 3 & 3 & - & - & - & - \\
\hline \multirow{6}{*}{\begin{tabular}{l}
0 \\
\multirow{2}{*}{} \\
1 \\
1 \\
$\omega$
\end{tabular}} & Early - 4 & 4 & 0 & Pos 1 & 1 & - & - & - & - \\
\hline & & & & Neg 3 & 3 & - & - & - & - \\
\hline & Moderate- 5 & 5 & 0 & Pos 4 & 1 & - & 2 & - & 1 \\
\hline & & & & Neg 1 & 1 & - & - & - & - \\
\hline & Advanced- 4 & 4 & 0 & Pos 4 & 1 & - & - & 1 & 2 \\
\hline & & & & Neg 0 & - & - & - & - & - \\
\hline
\end{tabular}

Table 3. Sensitivity, specificity, Positive and negative predictive value for each lesion Table 3.1. Leukoplakia

\begin{tabular}{lcccc}
\hline & \multicolumn{3}{c}{ Histopathological-16 } \\
\hline & & Dysplasia Positive-15 & Dysplasia Negative-6 & \\
Chemiluminescent Test & Positive-15 & 15 & 0 & Positive predictive \\
Outcome-21 & True Positive & False Positive & value $=1$ \\
& Negative-6 & 0 & 6 & Negative predictive \\
& & False Negative & True Negative & value $=1$ \\
& Sensitivity $=1$ & Specificity $=1$ & \\
\hline
\end{tabular}

Table 3.2. Oral Lichen Planus

\begin{tabular}{lcccc}
\hline & & \multicolumn{3}{c}{ Histopathological-16 } \\
\hline \\
Chemiluminescent Test & Positive- 5 & Dysplasia Positive-3 & Dysplasia Negative-13 & \\
Outcome-16 & 2 & 3 & Positive predictive \\
& Negative- 11 & True Positive & False Positive & value $=0.40$ \\
& & 1 & 10 & Negative predictive \\
& & False Negative & True Negative & value $=0.9$ \\
& Sensitivity $=0.67$ & Specificity $=0.77$ & \\
\hline
\end{tabular}


Table 3.3. Oral Sub mucous fibrosis

\begin{tabular}{|c|c|c|c|c|}
\hline \multicolumn{5}{|c|}{ Histopathological-13 } \\
\hline & & Dysplasia Positive-6 & Dysplasia Negative-7 & \\
\hline \multirow[t]{3}{*}{$\begin{array}{l}\text { Chemiluminescent Test } \\
\text { Outcome } 13\end{array}$} & Positive- 9 & $\begin{array}{c}6 \\
\text { True Positive }\end{array}$ & $\begin{array}{c}3 \\
\text { False Positive }\end{array}$ & $\begin{array}{l}\text { Positive predictive } \\
\text { value }=0.67\end{array}$ \\
\hline & Negative- 4 & $\begin{array}{c}0 \\
\text { False Negative }\end{array}$ & $\begin{array}{c}4 \\
\text { True Negative }\end{array}$ & $\begin{array}{l}\text { Negative predictive } \\
\text { value }=1\end{array}$ \\
\hline & & Sensitivity $=1$ & Specificity $=0.57$ & \\
\hline \multicolumn{5}{|c|}{ Histopathological-50 } \\
\hline \multirow{4}{*}{$\begin{array}{l}\text { Chemiluminescent Test } \\
\text { Outcome } 50\end{array}$} & & Dysplasia Positive-24 & Dysplasia Negative-26 & \\
\hline & Positive- 29 & $\begin{array}{l}23 \\
\text { True Positive }\end{array}$ & $\begin{array}{c}6 \\
\text { False Positive }\end{array}$ & $\begin{array}{l}\text { Positive predictive } \\
\text { value }=0.79\end{array}$ \\
\hline & Negative- 21 & $\begin{array}{c}1 \\
\text { False Negative }\end{array}$ & $\begin{array}{c}20 \\
\text { True Negative }\end{array}$ & $\begin{array}{l}\text { Negative predictive } \\
\quad \text { value }=0.95\end{array}$ \\
\hline & & Sensitivity $=0.96$ & Specificity $=0.77$ & \\
\hline
\end{tabular}

Table 4. Test values

\begin{tabular}{lccc}
\hline Parameters & Leukoplakia & Oral Lichen Planus & Oral Sub Mucous Fibrosis \\
\hline Sensitivity & 1 & 0.67 & 1 \\
Specificity & 1 & 0.77 & 0.57 \\
Positive Predictive Value & 1 & 0.4 & 0.67 \\
Negative Predictive Value & 1 & 0.9 & 1 \\
Accuracy & 1 & 0.75 & 0.77 \\
F1 score & 1 & 0.5 & 0.80 \\
False Positive Rate & 0 & 0.33 & 0.43 \\
False Discovery Rate & 0 & 0.6 & 0.33 \\
\hline
\end{tabular}

an updated product called ViziLite Plus. The only difference between the two products is that the latter now includes another solution, similar to the metachromatic dye Toludiene Blue and this is used to mark the ViziLitepositive lesions ${ }^{15}$.

This study is $100 \%$ sensitive \& specific for Leukoplakia. After rinsing with acetic acid solution, the increased keratin accumulation becomes more evident and thus the, chemiluminescent technique appears more reliable. Awan ${ }^{16}$ stated that the device can be used as a general oral mucosal examination system and may in particular improve the visualization of leukoplakias but it does not accurately delineate dysplasias. By far an experienced clinician can also differentiate between hyperkeratotic components from a suspicious area. It would have been highly appreciated, if by using vizilite, a new suspicious area was found other than what was observed clinically.
In this study, it was noted that there were no new lesions detected. This is in concordance with Mehrotra et al ${ }^{14}$ who stated that the light based oral cancer screening aids do not add any benefits to a conventional screening examination involving the use of a standard overhead light.

With relation to Oral Lichen Planus, the sensitivity was just $67 \%$ while the specificity was $77 \%$. Positive Predictive value was very less to about four. This can be attributable to the 3 false positive cases, 2 being reticular and one erosive. Type I error is high, resulting in low specificity. The false positivity could be due to the fact that keratotic component of oral lichen planus is less and the optical reflectance of the Vizilite could not be appreciated. In a study by Ram et $\mathrm{al}^{9}$, all the five clinically identified and histologically confirmed cases of oral lichen planus that were positive with Vizilite were considered as false positives. In the 10 cases of OSMF analysed, it was observed 
that all are males and the positive predictive value was only 0.67 . However, the negative predictive value was 1 . All the cases were true negatives. So, Vizilite is not reliable for diagnosing dysplastic lesions in oral lichen planus and OSMF.

The efficacy of Vizilite was tested by different studies. Huber ${ }^{11}$ stated that epithelium with hyperkeratinisation, hyperparakeratinisation, chronic inflammatory infiltrate, and an increase in the nuclear to cytoplasmic ratio reflect the diffuse, low-level, blue-white chemiluminescent light more strongly and appear "aceto-white". These bright white lesions are sharply demarcated from adjacent, normal epithelium, which takes on a blue hue. But in another study by Ram et $\mathrm{al}^{9}$, it was observed that lesions ranging from benign keratoses, through to oral lichen planus and oral squamous cell carcinoma tested positive with ViziLite. The accuracy of ViziLite in another study by Farah et $\mathrm{al}^{15}$ was only $18.2 \%$. Oh and Laskin ${ }^{17}$ screened about 100 patients with Vizilite and concluded that, although using an acetic acid rinse may have some benefit in making mucosal changes more visible, there appears to be no added benefit from using a chemiluminescent light rather than incandescent light for subsequent oral examination. Also, it stated that the chemiluminescent light produced reflections that made visualization more difficult and thus was not beneficial. In the present study, the overall sensitivity and specificity of Vizilite (Table 3.4 ) is 0.96 and 0.77 respectively. The Positive Predictive Value is only $79.31 \%$, while the Negative Predictive Value is $95.23 \%$. If Leukoplakia was not included, the values would have been still lesser. Analysis of other studies and correlating the same with the present study, gives us the impression that Vizilite, a costly clinical adjunct to diagnosis neither aid in better diagnosis of oral lesions nor in diagnosing dysplastic sites suitable for biopsy. The chance of false positivity is more which further distress the patients. Kujan ${ }^{18}$ stated that reducing oral cancer mortality and morbidity and improving the quality of life remain the primary goal and in the time to find an appropriate adjunct reliable tool for oral cancer screening, conventional oral examination constitutes the gold standard screening tool for potentially malignant oral lesions and cancer.

In conclusion, mere visualization of the oral cavity without identification of new lesions questions the use of Vizilite. In addition it does not differentiate between keratotic, inflammatory, malignant or Oral Potentially Malignant Disorders. There is no alteration in the biopsy site, after examination with Vizilite from that decided through complete examination by an Oral Medicine expert.There is no profound use of Vizilite in an already diagnosed oral premalignant lesion. Moreover, no additional lesions were detected by Vizilite. So, appropriate, systematic, competent clinical examination and scalpel biopsy definitely aids in diagnosis and subsequent management of oral mucosal lesions than Vizilite.

\section{References}

1. Jemal A, Bray F, Melissa M. Center, Jacques Ferlay, Elizabeth Ward, David Forman. Global Cancer Statistics. Ca Cancer J Clin. 2011; 61:69-90.

2. Mashberg A, Samit A. Early Diagnosis of Asymptomatic Oral and Oropharyngeal Squamous Cancers.Ca Cancer J Clin. 1995; 45:328-51.

3. Neville BW, Day TA. Oral Cancer and Precancerous Lesions. Ca Cancer J Clin. 2002; 52:195-215.

4. George A, Sreenivasan BS, Sunil S, Varghese SS, Thomas J, Gopakumar D, Mani V. Potentially malignant disorders of oral cavity. Oral Maxillofac Pathol J. 2011; 2:95-100.

5. Warnakulasuriya S, Johnson NW, Waal IV. Nomenclature and classification of potentially malignant disorders of the oral mucosa. J Oral Pathol Med. 2007; 36:575-80.

6. Slaughter M, Danely P, Harry W, Southwick M, Smejkal W. Field cancerization in oral stratified squamous epithelium clinical implications of multicentric origin. Cancer. 1953; 6:963-8.

7. Guggenheimer J, Verbin RS, Johnson JT, Horkowitz CA, Myers EN. Factors Delaying the Diagnosis of Oral and Oropharyngeal Carcinomas. Cancer. 1989; 64:932-5.

8. Rao SVK, Mejia G, Roberts-Thomson K, Logan R. Epidemiology of Oral Cancer in Asia in the Past DecadeAn Update (2000-2012). Asian Pac J Cancer Prev. 2013; 14 (10):5567-77.

9. Ram S, Siar CH. Chemiluminescence as a diagnostic aid in the detection of oral cancer and potentially malignant epithelial lesions. Int J Oral Maxillofac Surg. 2005; 34:521-7.

10. Lonky NM, Mann WJ, Massad LS, Mutch DG, Blanco JS, Vasilev SA. Ability of visual tests to predict underlying cervical neoplasia: colposcopy and speculoscopy. J Reprod Me. 1995; 40:530-6.

11. Huber MA, Bsoul SA, Terezhalmy GT. Acetic acid wash and chemiluminescent illumination as an adjunct to conventional oral soft tissue examination for the detection of dysplasia: a pilot study. Quintessence Int. 2004; 35(5): 378-84.

12 Epstein JB, Gorsky M, Lonky S, Silverman Jr S, Epstein JD, Bride M. The efficacy of oral lumenoscopy (ViziLite) in visualizing oral mucosal lesions. Spec Care Dent. 2006; 26(4):171-4. 
13. Kerr AR, Sirois DA, Epstein JB. Clinical evaluation of chemiluminescent lighting: an adjunct for oral mucosal examinations. J Clin Dent. 2006; 17(3):59-63.

14. Mehrotra R, Singh M, Thomas S, Nair P, Pandya S, Nigam NS, Shukla P. A cross-sectional study evaluating chemiluminescence and autofluorescence in the detection of clinically innocuous precancerous and cancerous oral lesions. J Am Dent Assoc 2010; 141:151-6.

15. Farah CS, McCullough MJ. A pilot case control study on the efficacy of acetic acid wash and chemiluminescent illumination (ViziLite trade mark) in the visualisation of oral mucosal white lesions. Oral Oncol. 2007; 43(8):820-4.

16. Awan KH, Morgan PR, Warnakulasuriya S. Utility of chemiluminescence (ViziLite ${ }^{\mathrm{Tx}}$ ) in the detection of oral potentially malignant disorders and benign keratoses. J Oral Pathol Med. 2011; 40:541-4.

17. Oh ES, Laskin DM. Efficacy of the ViziLite system in the identification of oral lesions. J Oral Maxillofac Surg. 2007; 65(3):424-6.

18. Kujan O, Sloan P. Dilemmas of Oral Cancer Screening: An Update. Asian Pacific J Cancer Prev. 2013; 14(5):3369-73. 